\title{
Tuneable superradiant thermal emitter assembly
}

\author{
Sudaraka Mallawaarachchi* and Malin Premaratne ${ }^{\dagger}$ \\ Advanced Computing and Simulation Laboratory $(A \chi L)$, Department of Electrical and Computer Systems Engineering, Monash University, \\ Clayton, Victoria 3800, Australia \\ Sarath D. Gunapala \\ Jet Propulsion Laboratory, California Institute of Technology, Pasadena, California 91109, USA \\ Philip K. Maini \\ Centre for Mathematical Biology, Mathematical Institute, Oxford University, 24-29 St. Giles', Oxford OX1 3LB, United Kingdom
}

(Received 11 January 2017; published 25 April 2017)

\begin{abstract}
Superradiance is a signature effect in quantum photonics that explains the collective enhancement of emission power by a factor of $N^{2}$ when $N$ emitters are placed in subwavelength proximity. Although the effect is inherently transient, successful attempts have been made to sustain it in the steady-state regime. Until recently, the effects of superradiance were not considered to be applicable to thermal emitters due to their intrinsic incoherent nature. Novel nanophotonic thermal emitters display favorable coherent characteristics that enable them to obey principles of superradiance. However, published analytical work on conventional superradiant thermal emitter assemblies shows an anomalous power scaling of $1 / N$, and therefore increasing the number of thermal emitters leads to a degeneration of power at resonance. This phenomenon immediately renders the effect of thermal superradiance futile since it cannot outperform noncoupled emitters in the steady-state regime. We propose an alternative assembly of thermal emitters with specific features that improves the power scaling while maintaining the effects of superradiance. In essence, we show that our emitter assembly achieves superior power delivery over conventional noncoupled emitter systems at resonance. Additionally, this assembly has the ability to be tuned to operate at specific resonant frequencies, which is a vital requirement for applications such as photothermal cancer therapy.
\end{abstract}

DOI: 10.1103/PhysRevB.95.155443

\section{INTRODUCTION}

Superradiance, a phenomenon conceptualized by Dicke in 1954 [1], is a fundamental theory in quantum photonics that has undergone rigorous studies for several decades [2-4]. It was originally designated to explain the ephemeral, enhanced, cooperative spontaneous emission characteristics of excited quantum emitters that are coherently coupled within a subwavelength proximity. Due to time-reversal symmetry, the same concept can be extended to describe its absorption counterpart known as superabsorption [5].

Recently, many efforts have been made to sustain the effects of superradiance in the steady-state regime for better utilization in applications [6-9]. One major breakthrough in these efforts is the designing and development of the superradiant laser [10]. Several mechanisms are being used to achieve steady-state superradiance. One such method is through the application of quantum feedback control, which involves regulating the number of excitons within different quantum states of the Dicke ladder [11]. This is realized through the careful designing of a photonic cavity or a band-gap crystal that can eliminate undesirable transitions, thus sustaining the effects of superradiance in the steady-state regime [5].

Until recently, the analog effect of thermal superradiance was not a topic of interest due to the incoherent nature of bulk thermal emitters $[12,13]$. However, nanophotonic emitters

\footnotetext{
*sudaraka@ieee.org

†malin.premaratne@monash.edu
}

show interesting coherent and enhanced thermal properties that compel them to acquiesce to the principles of superradiance [14-20]. Beyond displaying coherent characteristics, nanophotonic thermal emitters also possess the ability to radiate beyond the traditional blackbody limit [21,22].

Recently, Zhou et al. [23] have studied the superradiant characteristics of a conventional thermal emitter assembly where all emitters are identical and possess similar absorption $\left(\gamma_{a}\right)$ and far-field coupling $\left(\gamma_{c}\right)$ characteristics. For such an assembly, when the emitters obey the concept of optical resonance and are placed within subwavelength dimensions, superradiant phenomena can be observed at a controllable resonance frequency as long as the overcoupling relationship $\left(\gamma_{c} \gg \gamma_{a}\right)$ is maintained.

However, the subwavelength emitter placement requirement is difficult to achieve using conventional dielectric resonators because their dimensions exceed required criteria. Therefore, the conventional emitter assembly in [23] assumes that all emitters possess ultracompact electromagnetic resonance features. In practice, these resonance features can be generated using perfect electric conductor (PEC) deep subwavelength slits [24].

The analysis by Zhou et al. [23] shows that the effects of thermal superradiance vary drastically from the effects of the quantum counterpart. While the transient, ephemeral character of the effect is unaltered, it introduces significant spectral broadening. For conventional assemblies, they prove that the emission power at resonance is scaled inversely as a function of the number of emitters in the assembly. Due to this anomalous phenomenon, conventional superradiant thermal 


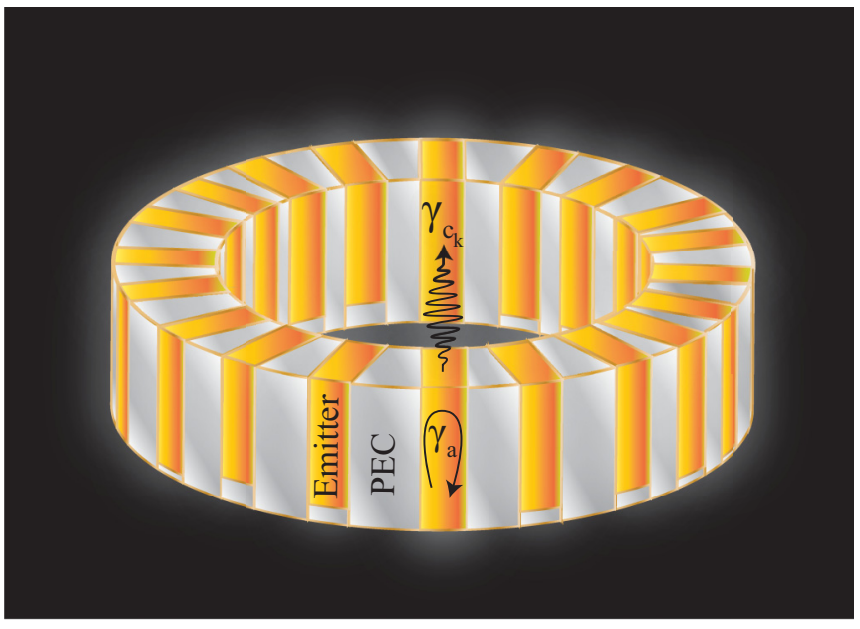

FIG. 1. Conceptual design for a ring-shaped superradiant emitter assembly with twenty nanophotonic emitters placed within subwavelength proximity. This structure of the emitter assembly can be engineered using PEC deep subwavelength slits. The absorption rate of the emissive material within each nanophotonic emitter is $\gamma_{a}$ and the far field coupling constant of the $k$ th emitter is $\gamma_{c_{k}}$. Note that because the emitters in the assembly have different dimensions, some emitters do not traverse the entire thickness of the PEC material, thus resulting in a gap between the bottom surface of the ring and the emitter.

emitter assemblies always fail to outperform noncoupled emitter assemblies at resonance, and this curtails the practical applicability of thermal superradiance.

In this paper, we focus on providing a solution to the spectral broadening phenomenon by considering an emitter assembly (see Fig. 1) comprising emitters with diverse farfield coupling constant characteristics. This emitter assembly can be constructed using an arbitrary number of emitters depending on the requirement that the subwavelength emitter placement criterion not be violated.

First we derive analytical solutions to the emission cross section and the power spectral density for our assembly. Our results are more generalized than what is being reported in [23]. Therefore, as a special case, we can produce the results in [23] by assuming an emitter assembly of identical emitters, thus providing a strong validity test for our results. However, the relaxation of the identical emitter assumption leads to undesirable alterations to resonant frequencies of the emitters. If not addressed, this alteration will hinder the performance of the proposed emitter configuration entirely. To overcome this, we suggest an emitter dimension tuning approach to maintain superradiance at a preselected mode frequency.

In Sec. II A of this paper, we present the analytical framework for deriving the cross section and power spectral density of our emitter assembly model. In Sec. II B, we reenforce identical emitter conditions and compare the derivations with those in [23]. Then in Sec. II C, we focus on addressing the resonance frequency tuning issue faced by our emitter assembly design and provide a flexible solution. Then an analysis is carried out in Sec. II D to prove that our emitter assembly can indeed outperform noncoupled emitter assemblies in the steady-state regime. Finally we present our analytical results in Sec. III A and discuss a possible application of the proposed superradiance assembly to photothermal cancer therapy in Sec. III B.

\section{ANALYTICAL FRAMEWORK}

\section{A. Generalized emitter assembly model}

The following analysis based on coupled-mode theory $[25,26]$ facilitates the studying of the intrinsic electromagnetic fields of the proposed emitter array. Let $\boldsymbol{a}_{\omega, t}=$ $\left[a_{1}, a_{2}, \ldots, a_{N}\right]^{T}$ denote the normalized electric field amplitudes within the emitters where $E_{k}=\left|a_{k}\right|^{2}$ is the energy in the $k$ th emitter. For an assembly of $N$ emitters, their time-varying amplitudes are governed by the following equation [27]:

$$
\frac{\partial}{\partial t} \boldsymbol{a}_{\omega, t}=\left[\left(\mathrm{j} \omega_{0}-\frac{\gamma_{a}}{2}\right) \boldsymbol{I}-\boldsymbol{\Gamma}_{\boldsymbol{c}}-\boldsymbol{\Gamma}_{\chi}\right] \boldsymbol{a}_{\omega, t}+\sqrt{\gamma_{a}} \boldsymbol{n},
$$

where $\boldsymbol{I}$ is the $N \times N$ identity matrix, $\omega_{0}$ is the resonant frequency of all emitters, and $j$ is the imaginary unit. Furthermore, the fluctuation dissipation theorem [28] requires absorption processes of this setup to be supplemented by the introduction of random thermal sources denoted by the $1 \times N$ matrix: $\boldsymbol{n}$. These thermal sources satisfy the following relationship [29]:

$$
\left\langle n_{i}^{*}(\omega) n_{j}\left(\omega^{\prime}\right)\right\rangle=\frac{\Theta_{\omega T}}{2 \pi} \delta\left(\omega-\omega^{\prime}\right) \delta_{i, j},
$$

where $\Theta_{\omega T}=\hbar \omega /\left(e^{\frac{\hbar \omega}{k_{B} T}}-1\right)$ represents the total energy of thermal photons generated at temperature $T$ at an angular frequency $\omega$ while $\hbar, k_{B}, \delta\left(\omega-\omega^{\prime}\right)$, and $\delta_{i, j}$ represent the reduced Planck constant, Boltzmann constant, Dirac delta function, and Kronecker delta function, respectively. This relationship will be required at the end of this section to obtain an expression for the emission cross section of the proposed emitter assembly.

For any emitter in the assembly, amplitude suppression can occur through the decaying of a generated photon. A photon may decay as a result of coupling to the far field or by being absorbed within the emitter itself. Let $\gamma_{c_{k}}$ denote the coupling rate at which a photon generated in the $k$ th emitter couples to the far field and $\gamma_{a}$ denote the rate at which a photon is absorbed by the absorptive dielectric material within the emitter itself. As observed in Eq. (1), it is assumed that $\gamma_{a}$ is constant for the entire emitter array and the far field coupling matrix $\boldsymbol{\Gamma}_{\boldsymbol{c}}$ is given by

$$
\boldsymbol{\Gamma}_{\boldsymbol{c}}=\frac{1}{2} \operatorname{diag}\left[\gamma_{c_{1}} \ldots \gamma_{c_{N}}\right]_{N \times N} .
$$

The effects of superradiance are governed by the presence of inter-resonant-emitter indirect interactions denoted by $\boldsymbol{\Gamma}_{\chi}$, the analytical form of which is obtained by quantifying the coupling effects between the $k$ th emitter and free space.

In order to model free space, we use the temporal coupled-mode theory based approach suggested by Verslegers et al. [30]. In this approach, free space is assumed to comprise an infinite number of modes and fragment into an infinite number of resonating Fabry-Perot cavity based radiation channels. Depending on the type of metal used for emitter design, plasmonic-mode channels may also appear. However for this analysis, we assume that the emitter assembly is constructed using nonplasmonic metals and neglect this phenomenon. This is a safe assumption for PECs due to their infinite-permittivity characteristics that prevent them from 


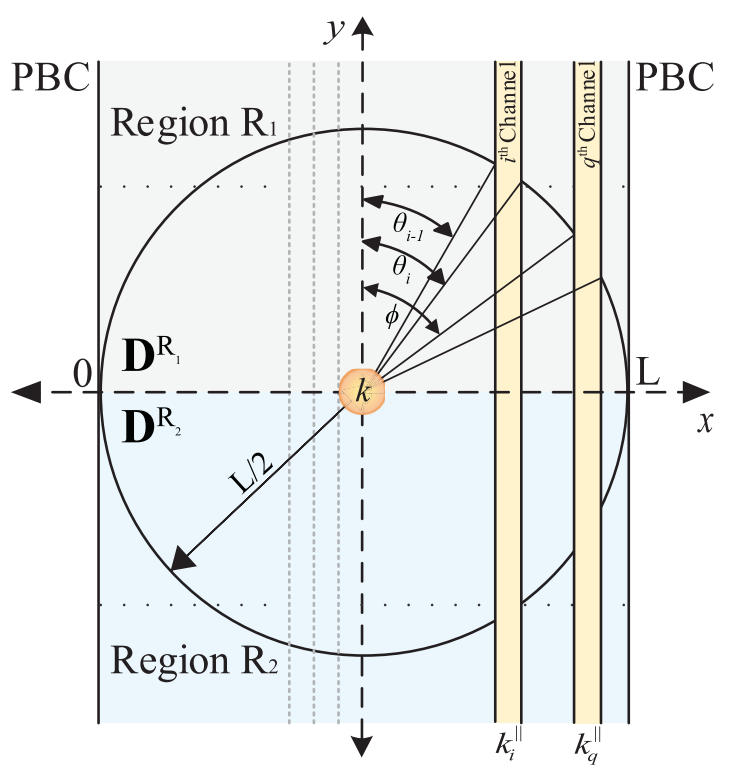

FIG. 2. Diagram illustrating the vertical distribution of normalized and orthogonal radiation channels. The $k$ th emitter is located at the midpoint between the periodic boundaries located a distance of $L$ apart. The infinite reality of free space is achieved by considering $L \rightarrow \infty$.

creating surface plasmons [31] and can be safely extended to some metals when the emissions occur at higher optical frequencies [32-34].

Furthermore, modal analysis is used to prove that the plane wave propagating channels should be orthogonal [25]. Based on these assumptions and proofs, the 2-dimensional free space is modeled using a set of orthogonal and normalized radiation channels denoted by $S$, where $P_{i}=\left|S_{i}\right|^{2}$ denotes the power of the $i$ th channel.

In order to deal with the infinite nature of free space, a periodic boundary condition (PBC) of length $L$ is introduced along the $x$ direction such that $L / \lambda_{0} \in \mathbb{Z}^{+}$, where $\lambda_{0}$ is the resonant wavelength. Enforcing $L \rightarrow \infty$ enables the recovery of free-space conditions. The $i$ th channel propagates a plane wave represented by the parallel component of the wave vector $\mathcal{K}_{i}^{\|}=i\left(2 \pi / m \lambda_{0}\right)$, where $m=L / \lambda_{0}$. In this model, there are $2 m$ radiation channels enclosed within the periodic boundary and each channel is uniquely represented by its index $i=$ $(-m,-m+1, \ldots,-1,1, \ldots, m-1, m)$. Each channel has a spatial width of $\lambda_{0} / 2$, which implies that the orthogonal set of channels uniformly sample the parallel wave vector space as shown in Fig. 2.

Overall emission to free space from all emitters can be defined using the following relationship:

$$
\boldsymbol{S}=\left[\begin{array}{l}
\boldsymbol{D}^{R_{1}} \\
\boldsymbol{D}^{R_{2}}
\end{array}\right] \boldsymbol{a}=\boldsymbol{D} \boldsymbol{a},
$$

where $\boldsymbol{D}^{R_{1}}$ and $\boldsymbol{D}^{R_{2}}$ are of dimensions $2 m \times N$. Due to the symmetric nature of the two regions, it is observed that $D^{R_{1}}=$ $D^{R_{2}}$, and their elements $D_{i, k}^{R_{1}}$ and $D_{i, k}^{R_{2}}$ represent the coupling of the $k$ th emitter to the $i$ th channel in regions $R_{1}$ and $R_{2}$, respectively.
Next we derive an analytical solution for $\boldsymbol{D}$ by considering the coupling from the $k$ th emitter to the free space. We begin by analyzing the geometry in Fig. 2 . We assume that the $k$ th emitter of the assembly placed at the center has an isotropic radiation characteristic. Using the definition for $\operatorname{arc}\left(\Delta \theta_{i}\right)=$ $\operatorname{arc}\left(\theta_{i}-\theta_{i-1}\right)=(L / 2) \Delta \theta_{i}$, the following result can easily be proven using simple trigonometric relations:

$$
\text { as } \begin{aligned}
L \rightarrow \infty, \cos \theta_{i} & =\frac{\lambda_{0} / 2}{\operatorname{arc}\left(\Delta \theta_{i}\right)}, \\
m \Delta \theta_{i} & =\frac{1}{\cos \theta_{i}} .
\end{aligned}
$$

The normal projection of the coupling components of the $k$ th emitter to the $i$ th channel can be expressed as follows:

$$
\gamma_{c_{\perp \leftarrow k}}=\gamma_{c_{\theta_{i} \leftarrow k}} \cos \theta_{i},
$$

where $\gamma_{c_{\theta_{j} \leftarrow k}}$ denotes the coupling from the $k$ th emitter along the $\theta_{i}$ direction and $\gamma_{c_{\perp \leftarrow k}}$ denotes the coupling component of the $k$ th emitter along the normal direction (parallel to the $y$ axis).

Based on the relationship in (6), we obtain the following equation for couplings from the $k$ th emitter to all channels in both regions $R_{1}$ and $R_{2}$,

$$
\gamma_{c_{k}}=2 \sum_{\substack{i=-m \\ m \neq 0}}^{m} \gamma_{c_{\theta_{i} \leftarrow k}}=2 \gamma_{c_{\perp \leftarrow k}} \sum_{\substack{i=-m \\ m \neq 0}}^{m} \frac{1}{\cos \theta_{i}} .
$$

By applying the summation condition to (5b) and considering either of the two symmetric regions $\left(R_{1}\right.$ or $\left.R_{2}\right)$, the following can be derived to simplify (7):

$$
\sum_{\substack{i=-m \\ m \neq 0}}^{m} \frac{1}{\cos \theta_{i}}=m \sum_{\substack{i=-m \\ m \neq 0}}^{m} \Delta \theta_{i}=m \pi,
$$

where $\theta_{i} \in(0, \pi / 2)$. Substituting the result from (8) in (7), the following answer is derived,

$$
\gamma_{c_{k}}=2 \gamma_{c_{\perp \leftarrow k}} m \pi
$$

The coupling element can now be written using the results from (6) and (9) as follows:

$$
D_{i, k}^{R_{1}}=D_{i, k}^{R_{2}}=\sqrt{\gamma_{c_{i} \leftarrow k}}=\sqrt{\frac{\gamma_{c_{k}}}{2 m \pi \cos \theta_{i}}},
$$

which allows us to explicitly generate the following:

$$
\boldsymbol{D}^{R_{1}}=\boldsymbol{D}^{R_{2}}=\left[\begin{array}{ccc}
\sqrt{\frac{\gamma_{c_{1}}}{2 m \pi \cos \theta_{-m}}} & \cdots & \sqrt{\frac{\gamma_{c_{N}}}{2 m \pi \cos \theta_{-m}}} \\
\vdots & \ddots & \vdots \\
\sqrt{\frac{\gamma_{c_{1}}}{2 m \pi \cos \theta_{-1}}} & \cdots & \sqrt{\frac{\gamma_{c_{N}}}{2 m \pi \cos \theta_{-1}}} \\
\sqrt{\frac{\gamma_{c_{1}}}{2 m \pi \cos \theta_{1}}} & \cdots & \sqrt{\frac{\gamma_{c_{N}}}{2 m \pi \cos \theta_{1}}} \\
\vdots & \ddots & \vdots \\
\sqrt{\frac{\gamma_{c_{1}}}{2 m \pi \cos \theta_{m}}} & \cdots & \sqrt{\frac{\gamma_{c_{N}}}{2 m \pi \cos \theta_{m}}}
\end{array}\right]_{2 m \times N},
$$

which can be used to obtain the final form of $\boldsymbol{D}$ using the relationship in (4). 
In order to obtain an analytical expression for $\boldsymbol{\Gamma}_{\chi}$, we consider the power relationship with regard to the far field and inter-emitter coupling. In order to obey conservation laws, it is required to cease the generation of new thermal photons $(\boldsymbol{n}=0)$ and to cease the absorption of generated photons $\left(\gamma_{a}=0\right)$. These modifications are applied to (1) and used in the following derivations:

$$
\begin{aligned}
\frac{\partial}{\partial t} \boldsymbol{a}^{*} \boldsymbol{a}= & {\left[\frac{\partial}{\partial t} \boldsymbol{a}^{*}\right] \boldsymbol{a}+\boldsymbol{a}^{*}\left[\frac{\partial}{\partial t} \boldsymbol{a}\right], } \\
= & \boldsymbol{a}^{*}\left[-j \omega_{0} \boldsymbol{I}-\left(\boldsymbol{\Gamma}_{\boldsymbol{c}}+\boldsymbol{\Gamma}_{\chi}\right)\right] \boldsymbol{a} \\
& +\boldsymbol{a}^{*}\left[j \omega_{0} \boldsymbol{I}-\left(\boldsymbol{\Gamma}_{\boldsymbol{c}}+\boldsymbol{\Gamma}_{\chi}\right)\right] \boldsymbol{a}, \\
= & \boldsymbol{a}^{*}\left[-2\left(\boldsymbol{\Gamma}_{\boldsymbol{c}}+\boldsymbol{\Gamma}_{\chi}\right)\right] \boldsymbol{a} .
\end{aligned}
$$

We now apply the same principle to the free-space model in Fig. 2 and obtain the following conservation relation: $\frac{\partial}{\partial t} \boldsymbol{a}^{*} \boldsymbol{a}=$ $-S^{*} \boldsymbol{S}=-\boldsymbol{a}^{*} \boldsymbol{D}^{*} \boldsymbol{D} \boldsymbol{a}$. Comparing this result with (12c), the following relationship is derived:

$$
\boldsymbol{D}^{*} \boldsymbol{D}=2\left(\boldsymbol{\Gamma}_{\boldsymbol{c}}+\boldsymbol{\Gamma}_{\chi}\right) .
$$

Using values from (3) and (11), it is now possible to obtain an analytical solution for $\left[\Gamma_{\chi}\right]_{i, k}$ as follows:

$$
\left[\Gamma_{\chi}\right]_{i, k}=\frac{1}{2} \sqrt{\gamma_{c_{i}} \gamma_{c_{k}}}\left(1-\delta_{i, k}\right) .
$$

Finally, we derive an analytical equation for the emission cross section of the emitter by considering spectral relations. Considering the physical nature of coupling constants and emitter amplitudes, it is possible to simplify the relations using $\forall_{i, k}\left(a_{k}, D_{i, k}\right) \in \mathbb{R} \Rightarrow \boldsymbol{D}^{*}=\boldsymbol{D}^{T}$. Total power emission by all channels across the spectrum is then given by $\left\langle P_{t}\right\rangle=\left\langle S^{*} \boldsymbol{S}\right\rangle=$ $\left\langle\boldsymbol{a}^{T} \boldsymbol{D}^{T} \boldsymbol{D} \boldsymbol{a}\right\rangle$.

As shown in Fig. 2, now we consider the coupling of the $k$ th emitter to an arbitrary $q$ th channel radiating at an angle $\phi$ where the channel spectrum is given by

$$
\left\langle P_{t, \phi}^{q}\right\rangle=\sum_{i=1}^{N} \sum_{k=1}^{N}\left\langle a_{i}^{*}(t) D_{i, q}^{T} D_{q, k} a_{k}(t)\right\rangle .
$$

The value of $\sum_{i=1}^{N} \sum_{k=1}^{N} D_{i, q}^{T} D_{q, k}$ can be readily found using (11) as follows:

$$
\sum_{i=1}^{N} \sum_{k=1}^{N} D_{i, q}^{T} D_{q, k}=\sum_{i=1}^{N} \sum_{k=1}^{N} \frac{\sqrt{\gamma_{c_{i}} \gamma_{c_{k}}}}{2 m \pi \cos \phi}
$$

Assuming that the thermal source has a stationary characteristic given by $\frac{d}{d t}|n(t)|^{2}=0$, it is possible to obtain the following relationship [28,29]:

$$
\left\langle a_{i}^{*}(t) a_{k}(t)\right\rangle=\int_{0}^{\infty} d \omega \int_{0}^{\infty} d \omega^{\prime} A_{i, k},
$$

where $A_{i, k}=e^{-j\left(\omega-\omega^{\prime}\right) t}\left\langle a_{i}^{*}(\omega) a_{k}\left(\omega^{\prime}\right)\right\rangle$. Applying the results from (16) and (17) in (15) yields the following expression:

$$
\left\langle P_{t, \phi}^{q}\right\rangle=\sum_{i=1}^{N} \sum_{k=1}^{N} \int_{0}^{\infty} d \omega \int_{0}^{\infty} \frac{\sqrt{\gamma_{c_{i}} \gamma_{c_{k}}}}{2 m \pi \cos \phi} A_{i, k} d \omega^{\prime} .
$$

Now it is possible to compare the frequency spectrum relation for the total emission power of the channel: $\left\langle P_{t, \phi}^{q}\right\rangle=$ $\int_{0}^{\infty} P_{\omega, \phi}^{q} d \omega$, with the result obtained in (18). This allows us to explicitly obtain an expression for the channel power spectral density as follows:

$$
P_{\omega, \phi}^{q}=\sum_{i=1}^{N} \sum_{k=1}^{N} \int_{0}^{\infty} \frac{\sqrt{\gamma_{c_{i}} \gamma_{c_{k}}}}{2 m \pi \cos \phi} A_{i, k} d \omega^{\prime} .
$$

In order to solve (19) analytically, it is necessary to obtain an expression for $\left\langle a_{i}^{*}(\omega) a_{k}\left(\omega^{\prime}\right)\right\rangle$ using the relationship given for thermal sources in (2). This is readily achieved by considering the time-varying dependence of emitter amplitudes given by $\boldsymbol{a}_{\omega, t} \approx \boldsymbol{a}_{0} e^{j \omega t}$ where matrix $\boldsymbol{a}_{0}=\left[a_{0_{1}}, a_{0_{2}}, \ldots, a_{0_{N}}\right]^{T}$ denotes the absolute amplitudes of the emitters. Note that this is a valid assumption in the weakly coupled regime for lossless emitter assemblies [26]. This allows us to formulate the following relationship:

$$
\frac{\partial}{\partial t} \boldsymbol{a}_{\omega, t} \approx j \omega \boldsymbol{a}_{\omega, t}
$$

Substituting the result from (1) in (20) yields the following for $\boldsymbol{a}_{\omega, t}$ :

$$
\boldsymbol{a}_{\omega, t} \approx\left\{\left[j\left(\omega-\omega_{0}\right)+\frac{\gamma_{a}}{2}\right] \boldsymbol{I}+\boldsymbol{\Gamma}_{\boldsymbol{c}}+\boldsymbol{\Gamma}_{\chi}\right\}^{-1} \sqrt{\gamma_{a}} \boldsymbol{n} .
$$

The inverse portion of (21) can be obtained using the KenMiller lemma [35]. Application of this mathematical lemma is subject to the necessary condition $\operatorname{rank}\left(\boldsymbol{\Gamma}_{\boldsymbol{C}}+\boldsymbol{\Gamma}_{\chi}\right)=1$. Combining the general result in (13) with (11) allows us to obtain an expression for $\boldsymbol{\Gamma}_{\boldsymbol{C}}+\boldsymbol{\Gamma}_{\boldsymbol{\chi}}$, on which we can perform Gaussian-Jordan eliminations to obtain the row-canonical form of the matrix, which readily proves that the necessary rank condition is satisfied [36] and, therefore, the lemma is applicable.

Defining the inverse portion of (21) as $\mathcal{R}$ and applying the lemma yields the following:

$$
\mathcal{R}=\frac{1}{2(\mathcal{A}+j \Omega)(\mathcal{X}+j \Omega)}\left[(\mathcal{X}+j \Omega) \boldsymbol{I}-\boldsymbol{D}^{\boldsymbol{T}} \boldsymbol{D}\right],
$$

where $\Omega$ is the detuning parameter given by $\omega-\omega_{0}$ and

$$
\begin{aligned}
& \mathcal{A}=\frac{\gamma_{a}}{2}, \\
& \mathcal{X}=\mathcal{A}+\sum_{i=1}^{N} \frac{\gamma_{c_{i}}}{2} .
\end{aligned}
$$

The result in (22) can now be applied to $\boldsymbol{a}_{\omega, t} \approx \sqrt{\gamma_{a}} \mathcal{R} \boldsymbol{n}$ and substituted in (19) to obtain the following result:

$$
P_{\omega, \phi}^{q} \approx \sum_{i=1}^{N} \sum_{k=1}^{N} \int_{0}^{\infty} \frac{\sqrt{\gamma_{c_{i}} \gamma_{c_{k}}}}{2 m \pi \cos \phi} \gamma_{a} \boldsymbol{\mathcal { R }}_{i, u}^{*} \boldsymbol{\mathcal { R }}_{k, v} N_{i, k} d \omega^{\prime},
$$

where $N_{i, k}=e^{-j\left(\omega-\omega^{\prime}\right) t}\left\langle n_{i}^{*}(\omega) n_{k}\left(\omega^{\prime}\right)\right\rangle$. Substituting the values from (2) now yields the following:

$$
\begin{aligned}
P_{\omega, \phi}^{q} \approx & \frac{\Theta_{\omega T}}{2 \pi} \frac{\gamma_{a}}{2 m \pi \cos \phi} \sum_{i=1}^{N} \sum_{k=1}^{N} \sum_{u=1}^{N} \sum_{v=1}^{N} \int_{0}^{\infty} \mathcal{R}_{i, u}^{*} \mathcal{R}_{k, v} \\
& \times \delta\left(\omega-\omega^{\prime}\right) e^{-j\left(\omega-\omega^{\prime}\right) t} \sqrt{\gamma_{c_{i}} \gamma_{c_{k}}} \delta_{u, v} d \omega^{\prime} .
\end{aligned}
$$

In order to obtain an expression for the power spectrum of all channels, it is necessary to decouple the channel 
dependency in (25). This can be done by normalizing the total emitted power in the channel by the flux density of a single channel. This is a valid assumption for an isotropic radiator and the channel flux density is given by $\Theta_{\omega T} /[2 \pi L \cos \phi][23,37]$. The total emission cross section for the emitter assembly has the following form:

$$
\sigma_{\omega}=\int_{0}^{2 \pi} \frac{P_{\omega, \phi}^{q}}{\frac{\Theta_{\omega T}}{2 \pi} / L \cos \phi} d \phi
$$

which allows us to obtain the following relation for $\sigma_{\omega}$ :

$$
\sigma_{\omega} \approx \frac{2 \pi c}{\omega_{0}} \gamma_{a} \sum_{i=1}^{N} \sum_{k=1}^{N} \sum_{u=1}^{N} \boldsymbol{R}_{i, u}^{*} \boldsymbol{R}_{k, u} \sqrt{\gamma_{c_{i}} \gamma_{c_{k}}}
$$

where $c$ is the velocity of light in free space.

By substituting the values for $\boldsymbol{\mathcal { R }}_{k, u}$ and its complex conjugate (c.c.) from (22), it is possible to derive the final equation for the emission cross section of the emitter assembly as follows:

$$
\sigma_{\omega} \approx \frac{\pi c}{\omega_{0}}\left(\frac{\gamma_{a}}{2} \sum_{i=1}^{N} \gamma_{c_{i}}\right) \frac{\left\{4\left[\left(\frac{\gamma_{a}}{2}+\sum_{i=1}^{N} \frac{\gamma_{c_{i}}}{2}\right)^{2}+\left(\omega-\omega_{0}\right)^{2}\right]+\sum_{i=1}^{N}\left[\gamma_{c_{i}}^{2}-4 \gamma_{c_{i}}\left(\frac{\gamma_{a}}{2}+\sum_{j=1}^{N} \frac{\gamma_{c_{j}}}{2}\right)\right]+2 \sum_{i=1}^{N-1} \sum_{j=i+1}^{N}\left(\gamma_{c_{i}} \gamma_{c_{j}}\right)\right\}}{\left[\left(\frac{\gamma_{a}}{2}\right)^{2}+\left(\omega-\omega_{0}\right)^{2}\right]\left[\left(\frac{\gamma_{a}}{2}+\sum_{i=1}^{N} \frac{\gamma_{c_{i}}}{2}\right)^{2}+\left(\omega-\omega_{0}\right)^{2}\right]}
$$

This is the main analytical result derived in this paper.

The total emission power of the emitter assembly can now be obtained by multiplying the emission cross section in (28) with the blackbody spectral density $\mathrm{BB}_{\mathrm{psd}}$ [38], which yields

$$
P_{\omega}=\mathrm{BB}_{\mathrm{psd}} \sigma_{\omega}=\left(\frac{\omega^{4}}{8 \pi^{3} c^{3}} \Theta_{\omega, T}\right) \sigma_{\omega} .
$$

\section{B. Spacial case: Conventional emitter assembly}

Now we focus on proving that our generalized results are valid for the special case of emitter assemblies comprising identical emitters, such as the conventional model discussed in [23]. For this setting, all emitters have the same coupling constant value which we denote by $\gamma_{c}$. This assumption leads to the simplification of the expression for $\boldsymbol{\Gamma}_{c}=\left(\gamma_{c} / 2\right) \boldsymbol{I}$. Based on this simplification, we can obtain the following mathematical expressions that are required to reenforce this assumption to our emitter assembly,

$$
\begin{gathered}
\sum_{i=1}^{N} \gamma_{c_{i}}=N \gamma_{c}, \\
2 \sum_{i=1}^{N-1} \sum_{j=i+1}^{N}\left[\gamma_{c_{i}} \gamma_{c_{j}}\right]=N(N-1) \gamma_{c}^{2} .
\end{gathered}
$$

Now these two simplifications are applied to (28), which yields the cross section for the conventional model as follows:

$$
\sigma_{\omega} \approx \frac{2 \pi c}{\omega_{0}} \frac{N \gamma_{a} \gamma_{c}}{\left(\omega-\omega_{0}\right)^{2}+\left(\frac{N \gamma_{c}+\gamma_{a}}{2}\right)^{2}} .
$$

The result obtained in (31) is identical to the cross section given in [23], which proves the mathematical consistency of our calculations. This result can be substituted in (29) in order to obtain the total emission power relation given in [23].

The most significant result derived in [23] is the contrast between nonresonant and conventional resonant emitter assemblies. For nonresonant and superradiant emitter assemblies of $N$ emitters each, the total power emission at resonance $(\omega=$ $\left.\omega_{0}\right)$ can be obtained by applying the nonresonant condition $\left(\gamma_{a} \gg \gamma_{c}\right)$ and the superradiant condition $\left(\gamma_{c} \gg \gamma_{a}\right)$ in (31), which yield the following results:

$$
\begin{aligned}
{ }^{\text {nonresonant }} P_{\omega} & \approx \frac{2}{\pi} \Theta_{\omega, T} \frac{\gamma_{c}}{\gamma_{a}} N, \\
\text { superradiant } P_{\omega} & \approx \frac{2}{\pi} \Theta_{\omega, T} \frac{\gamma_{a}}{\gamma_{c}} \frac{1}{N} .
\end{aligned}
$$

It is observed that nonresonant emitter assemblies, as expected, show a linear power scaling at resonance whereas conventional superradiant emitter assemblies show an anomalous power scaling by a factor of $1 / N$.

\section{Tuning the resonance frequency}

Our emitter assembly assumes that all emitters within it have the same absorption constant $\gamma_{a}$. Within the weak-field regime, this can be achieved by using an emitter array made using the same emissive material, which ensures that all emitters will have the same dielectric constant [39]. However, the superradiant phenomenon, as observed in (14), depends solely on the far-field coupling rates of the emitters. Therefore, the success of our proposed assembly depends on the individual tunability of this parameter in each emitter. Furthermore, it is vital to maintain the emitter assembly in the overcoupling regime $\left(\frac{1}{N} \sum_{i=1}^{N} \gamma_{c_{k}} \gg \gamma_{a}\right)$ in order to sustain the superradiant effects [40]. While these might appear as straightforward requirements, several design challenges need to be overcome to achieve a successful realization.

The main issue is with the design of the individual emitter unit. For the 2-dimensional case, it can be assumed that an assembly of ideal Fabry-Perot cavity emitters with a constant height will have $\gamma_{c}$ values proportional to their widths [23]. However, if the slit width is varied, it is no longer possible to assume that the resonant frequency $\omega_{0}$ is constant to all emitters [41]. This detuning effect needs to be addressed in order to maintain the functionality of our emitter assembly.

For their emitter model, Zhou et al. [23] have used an ideal Fabry-Perot resonant cavity. As shown in Fig. 3(a) and Fig. 3(c), for an ideal Fabry-Perot cavity with 1-dimensional control, resonant frequencies are governed by the equation $f_{m}=m c / 2 n L$ where $c$ is the velocity of light in free space, $L$ is the cavity length, $n$ is the refractive index of the cavity, and $m$ is the resonant mode. The cavity resonant mode can directly be tuned by changing the length of the cavity. This means that 
(a)
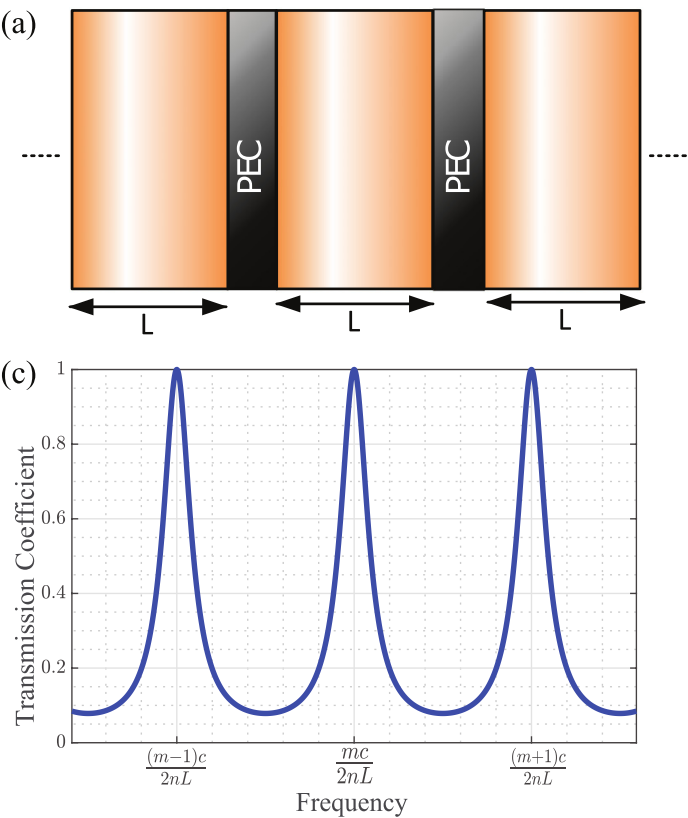

(b)

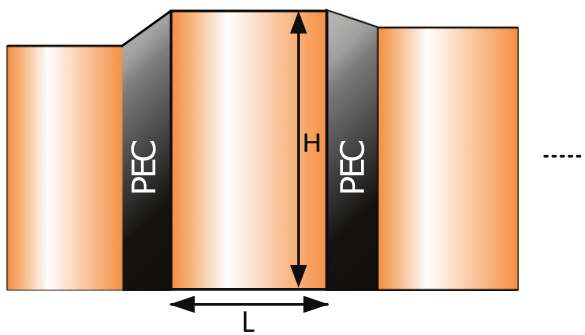

(d)

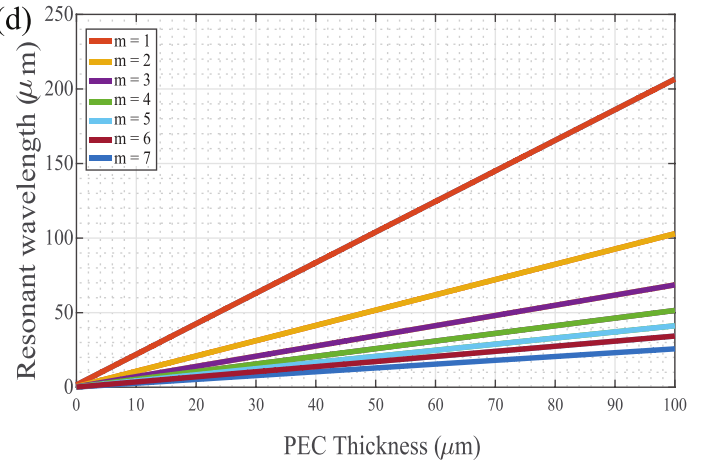

FIG. 3. (a) Emitter model used to design the conventional superradiant thermal emitter in [23]. (b) Emitter model used to design the tuneable emitter where $\omega_{0}$ is controlled by tunable PEC dimensions as shown. (c) Periodic resonant modes observed in a 1-dimensional Fabry-Perot emitter cavity. (d) Resonant modes of a tuneable cavity. Note the existence of multiple modes for the same PEC thickness and the linear relationship between PEC thickness and $\omega_{0}$.

for an ensemble of emitters with varying far-field coupling parameters, a unique resonant frequency cannot be maintained with 1-dimensional control. Therefore, this simplified emitter model fails to facilitate our design criteria.

Control of resonant frequency in metallic slits has been an area of interest for several decades $[41,42]$. While the problem itself is highly complex and analytical, within the weak-field regime, several simple relationships have been proposed to design cavities with arbitrary resonant frequencies. One such study [43] gives the following 2-dimensional control relationship for the resonant wavelength of a slit:

$$
\lambda_{m} \approx\left(\frac{2.05}{m} H+\frac{a}{m} L\right),
$$

where $a \approx 1.8+\pi \delta_{m, 1}$ and $L, H$ correspond to the cavity dimensions shown in Fig. 3(b). This relationship directly solves our design problem. The far-field coupling constant can now be tuned by changing the $L$ parameter and the resonant frequency can be tuned by changing the $H$ parameter as required. This clearly enables the retention of a unique resonant frequency by varying cavity dimensions. For a given thickness $(H)$, several resonant modes will exist as shown in Fig. 3(d).

Another challenge is associated with designing emitter arrays in such a manner as to avoid radiating into various far-field, non-superradiant modes. Failure to overcome this challenge suppresses the effects of superradiance and results in various uncontrollable phenomena. This problem has been studied both analytically and numerically by Verslegers et al. [40] for the case of 2 emitters. However, through their findings, it can be assumed that in general terms, maintaining the inter-emitter distances of the assembly within subwavelength dimensions is the sufficient criterion to avoid radiating into subradiant modes when operating in the overcoupling regime [23]. This observation agrees with the fact that all resonators operate under the same phase and therefore must exhibit qualities of superradiance, even at nonuniform overcoupling parameters across the emitters.

\section{Comparison with noncoupled emitters}

In the steady-state regime, emitters within noncoupled emitter assemblies behave independently. For an assembly of $N$ identical, noncoupled emitters, each with the ability to deliver power $P$ at resonance, the cumulative power delivery will be $N P$, a value that varies linearly with the number of emitters. In the steady-state regime, coupled emitter assemblies that obey the principles of superradiance have the potential to outperform noncoupled emitter assemblies. Assume a coupled emitter assembly of $N$ identical emitters, each with the ability to deliver power $P$ at resonance. In order to outperform the analogous noncoupled emitter assembly, the coupled emitter assembly must must maintain a power delivery greater than $P / N$ at resonance. In order to understand the power enhancement phenomenon, it is important to establish the power rate enhancement achievable through superradiance.

The concept of superradiance explains the enhancement of light-matter coupling observed in a set of dipole emitters positioned within subwavelength proximity and excited using an external electromagnetic field. These enhancements can be observed around the midpoint of the symmetric Dicke ladder [1]. Consider the case of $N$ emitters in subwavelength proximity. In these nanoscopic dimensions, light fails to distinguish between particles and thus interacts with the entire assembly collectively. Phenomena of this nature can 
be analyzed using collective operators

$$
\hat{J}_{ \pm}=\sum_{i=1}^{N} \hat{\sigma}_{ \pm}^{i}, \quad \hat{J}_{z}=\sum_{i=1}^{N} \hat{\sigma}_{z}^{i},
$$

where $\hat{\sigma}_{z}^{i}$ and $\hat{\sigma}_{ \pm}^{i}$ are the usual Pauli operators for the $i$ th dipole of a two-level system.

The light-matter interaction Hamiltonian is of the form shown in (35) and obeys SU(2) commutation relations,

$$
\hat{\mathcal{H}}_{\text {int }}=-\hat{E} \boldsymbol{d}\left(\hat{J}_{+}+\hat{J}_{-}\right),
$$

where $\hat{E}$ is the light field operator and $\boldsymbol{d}$ is the dipole matrix. This leads to a relationship involving Clebsch-Gordan coefficients that explains a system moving along ladder states characterized by

$$
\hat{J}_{ \pm}|L, M\rangle=\sqrt{(L \pm M+1)(L \mp M)}|L, M \pm 1\rangle,
$$

where $L$ and $M$ are eigenvalues of $\hat{J}^{2}$ and $\hat{J}_{z}$, respectively. The ladder transition rate for this scenario is given by

$$
\Gamma_{M \rightarrow M \pm 1}=\gamma\left(\frac{N}{2} \pm M+1\right)\left(\frac{N}{2} \mp M\right),
$$

where $\gamma$ denotes the decay rate of a free atom. The interesting observation here is at the midpoint of the ladder when $M=0$, for a large number of dipoles, Eq. (37) simplifies to

$$
\Gamma_{0 \rightarrow \pm 1} \approx \gamma\left(\frac{N}{2}\right)^{2}
$$

This quadratic dependence grasps the essence of superradiance: a transient, ephemeral effect where constructive interference enhances emission efficiency of emitters [44,45].
It was discussed in Sec. II B and proven in (32a) that nonresonant emitters show a linear power scaling at resonance. As shown in (32b), for conventional superradiant emitter configurations, the emission power scales anomalously by a factor of $1 / N$ at resonance during operation. It is seen in (38) that during sustained superradiance, it is possible to attain a temporal enhancement factor of $N^{2}$. Therefore, a conventional emitter assembly will only be able to show a power scaling of $1 / N \times N^{2}=N$ at resonance, which shows no improvement over nonresonant assemblies. Our assembly, as shown in Sec. III A, shows a power scaling of better than a factor of $1 / N$ at resonance and therefore has the ability to outperform nonresonant emitter assemblies at resonance.

\section{RESULTS AND DISCUSSION}

\section{A. Analytical results}

Figure 4(a) shows the power spectra obtained for the sample emitter assembly designs shown to the right. The design corresponding to Fig. 4(b) is the nonresonant emitter assembly case where the emitters are placed at a greater than subwavelength distance apart. The design shown in Fig. 4(f) corresponds to the conventional emitter model proposed in [23]. By comparing the spectrum curves obtained for the two designs, it is clear that the nonresonant emitter assembly result is in agreement with the linear power scaling proven in (32a) and the conventional superradiant configuration is in agreement with the anomalous power scaling proven in (32b).

Figures 4(c)-4(e) depict three possible emitter assembly configurations. The power spectral density curves based on (28) and (29) in this case show the expected enhancement by a factor better than $1 / N$. This is evident by the resonant peaks

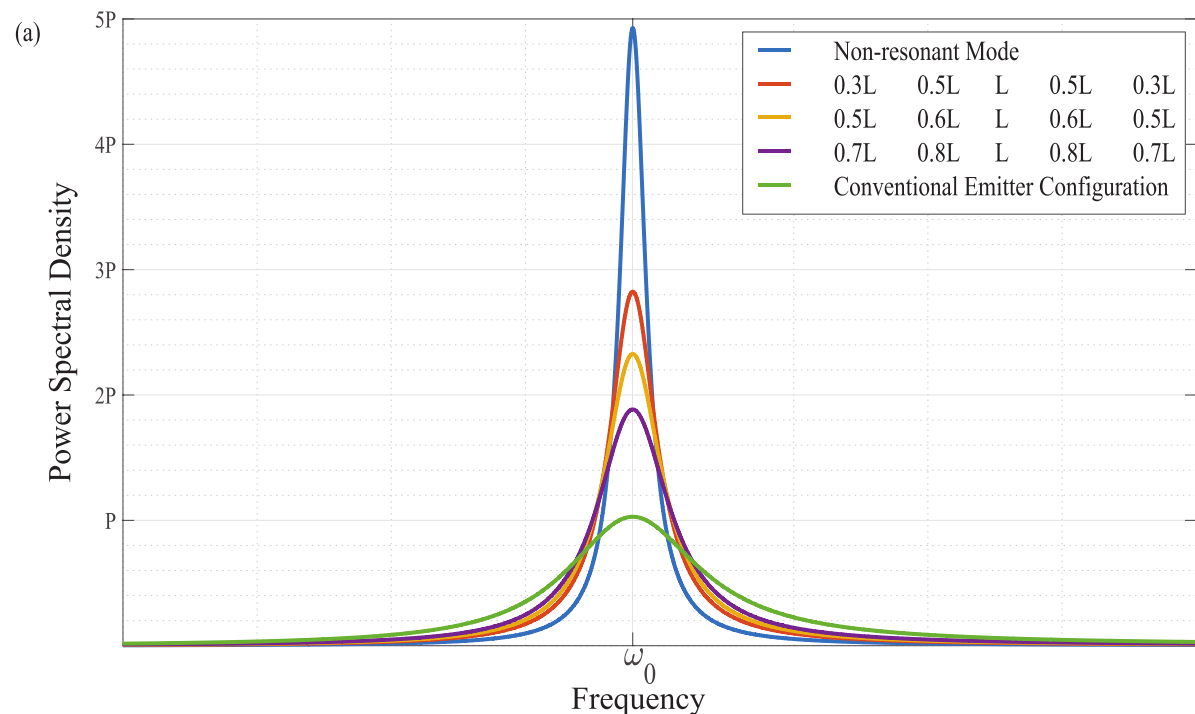

(b)

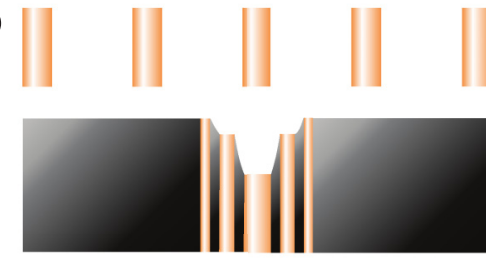

(d)

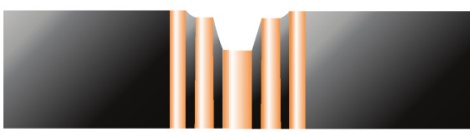

(e)

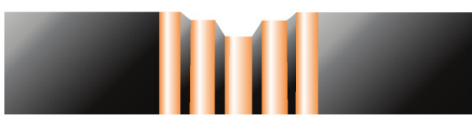

(f)

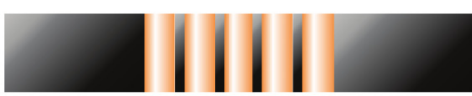

FIG. 4. (a) Power spectral density as a function of frequency for different emitter designs utilizing 5 emitters. Note how the power at $\omega_{0}$ varies for the different emitter configurations. The power scaling relationships for nonresonant and conventional superradiant modes agree with the direct results in (32a) and (32b). $P$ is the power spectral density of a noncoupled emitter with a width of $L$ and a thickness of $H$, corresponding to the dimensions of the middle emitter in each case. These symbolic values are chosen to represent typical values and exact, numerical values can be calculated using (29) for a given design. (b) Illustration of geometric distribution of nonresonant emitters. Note that the emitters are not in subwavelength proximity. (c)-(e) Configurations of tuneable superradiant emitters where the emitters are positioned in subwavelength proximity. (f) Emitter configuration for the conventional superradiant emitter case where the emitters are positioned in subwavelength proximity. All emitter dimensions are shown to scale. 
of these configurations being above the reference spectral value $P$. As described in Sec. IID, because of the contribution of the temporal superradiant phenomena, the overall potential power gain at the resonant frequency is now apparent. Note that all emitter dimensions are selected according to (33), a crucial criterion required to maintain the same mode at the same resonant frequency.

Through these results, it is evident that by relaxing the common coupling constant assumption for the emitter ensemble, it is possible to considerably alleviate the spectral broadening issues highlighted in [23]. As expected, the characteristic Lorentzian curve for the nonresonant mode still shows significantly less spectral broadening than all superradiant modes. However, this issue is not a theoretical concern. The goal of the emitter is to deliver the most amount of power at resonance. Upon achieving steady-state operation, the effects of superradiance will quadratically improve the emission efficiency as discussed in Sec. IID. While there are other losses that need to be considered when designing an operational emitter, any spectral suppression better than a factor of $1 / N$ should theoretically enhance the overall emission at resonance.

We have shown that by choosing a set of tuneable coupling constants, it is indeed possible to control the spectral suppression. In order to perform a fair comparison, we have scaled the coupling constants by tuning the dimensions with respect to the widest emitter in the array. In all of the emitter assembly designs illustrated in Figs. 4(b)-4(f), we have chosen the central emitter to be at the same, largest chosen width. Relative width scales of the other emitters are shown in Fig. 4(a). For the case of Figs. 4(b) and 4(f), all of the emitters have the same height and width. In other words, we have maintained symmetry around the central emitter in order to facilitate direct comparisons.

Note that none of these are theoretical limitations and these emitters can be tuned according to the design requirements. All emitters are filled with an emissive material with the same permittivity and $\gamma_{c_{i}}$ is taken as a quantity proportional to the emitter length as well as the permittivity of the emissive material [23].

\section{B. Proposed application: Photothermal cancer therapy}

Recently, there has been a keen interest in utilizing nanoscopic structures in cancer therapy. The minute nature of these devices theoretically enables minimally invasive treatment of cancers [46]. Therefore, various attempts are being made at understanding light, heat, and matter interactions [47], energy transportation [48-51], as well as the modeling of such nanoscopic devices targeting this application, ranging from gold nanoshells [52] to nanoscopic lasers known as spasers [53-55].

There are several key areas of significance when designing devices for photothermal cancer therapy. Increased power delivery is one such area that has been in the spotlight for several years [46]. As a solution, we propose the use of steady-state thermal superradiance as an enhanced power delivery tool for photothermal cancer therapy. To the best of our knowledge, this is the first theoretical formulation targeting the utilization of the principles of superradiance into enhancing this application. Another key area of interest with such devices is biocompatibility. Due to the tunable nature of our emitter assembly, it has the capability to be designed using a wide array of materials as suited for the application. Furthermore, applications of this nature require the maintenance of specific wavelengths to achieve optimal cellular ablation. Once again, the tunable nature of our design can be utilized for this purpose efficiently.

It is noted that the wavelength $1.45 \mu \mathrm{m}$ in the near-infrared spectrum is optimal for electromagnetic absorption by water, which will in turn be ideal for an application like photothermal cancer therapy [56]. Therefore, this value can be set as the resonant wavelength for a model superradiant emitter assembly.

As an example, consider using a PEC substrate with a slit thickness of $6 \mu \mathrm{m}$ for the first emitter of the assembly. The resonant frequency spectrum shown in Fig. 5 can now be obtained for the various possible modes for this thickness. From the spectrum, it is observed that the resonant wavelength is proximal to the 17 th mode. By using (33), now it is possible to solve for the other dimension of the cavity. In order to maintain superradiance without undergoing anomalous power suppression at resonance as shown in Fig. 4, now it is required to vary the thickness of the PEC substrate for the second emitter. This process can be followed iteratively until an emitter with suitable design characteristics is realized.

Design and realization of structures of nanoscopic magnitudes have been a subject of interest in recent times. Owing to the discovery of interesting characteristics of deep subwavelength slits, many attempts have been made towards

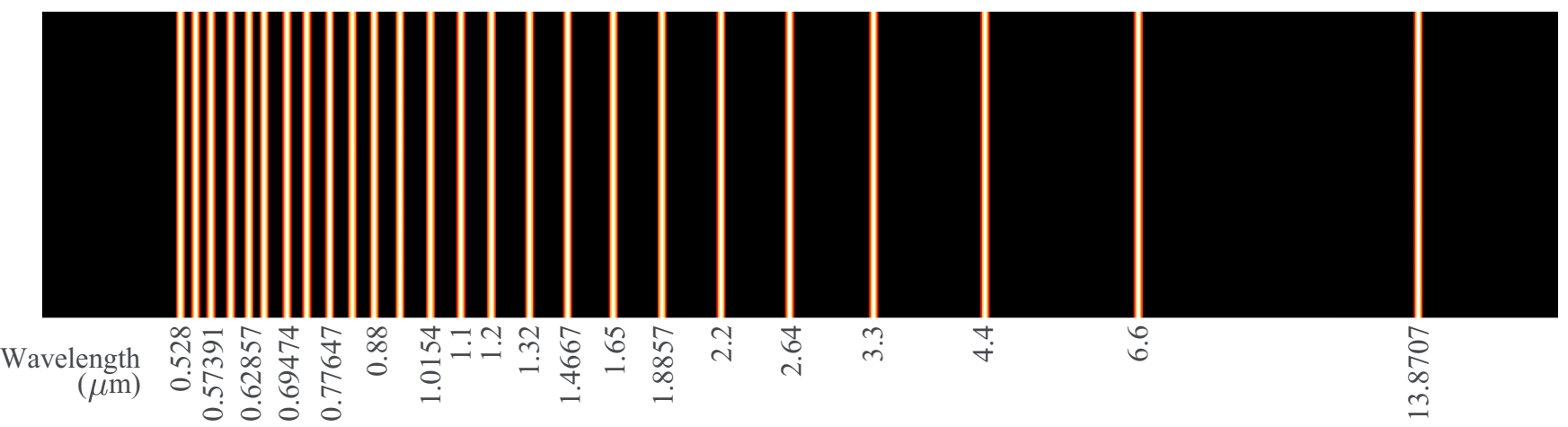

FIG. 5. Resonant spectrum of the first 25 modes for a single emitter designed using a PEC thickness of $6 \mu \mathrm{m}$. 
studying them experimentally. Since our design is entirely based on these deep subwavelength slits, the experimental feasibility is quite high. For instance, lithographic techniques utilizing focused-ion beams may be used with suitable masks to fabricate structures of this nature experimentally [57]. Furthermore, recent improvements in nanoscopic realization makes it possible to prototype nanoscale devices of a few orders of nanometers [58]. This further strengthens the experimental feasibility of our emitters, thus paving the way to assess the accuracy and relevance of the analytical results provided herein.

\section{CONCLUSION}

In this paper, we have obtained an analytical solution for the emission cross section and the power spectral density of a tuneable emitter design that shows superradiant characteristics. Originally, superradiance was not considered to be applicable to thermal emitters due to their incoherent emission properties. However, nanophotonic thermal emitters show coherent and enhanced emission properties, which make them ideal candidates to obey the principles of superradiance. Although superradiance is intrinsically an ephemeral and transient effect, many successful attempts have been made towards sustaining it in the steady-state regime.

Initial efforts at studying thermal superradiance using a conventional emitter model comprising emitters with identical absorption constants and far-field coupling constants showed that there is an anomalous power scaling at resonance. This observation completely renders the thermal superradiance phenomenon useless for practical applications due to the fact that such a system can never surpass noncoupled emitter assemblies in terms of power delivery at resonance. By relaxing the assumption of a constant far-field coupling rate for all emitters, we showed that it is possible to reduce the spectral broadening associated with the conventional assembly. This modification enables the utilization of thermal superradiance in a meaningful manner due to the possibility of enhanced power delivery over nonresonant assemblies at resonance. Furthermore, our tuneable emitter design can be realized to operate at a predefined resonant frequency for a specific application. Through this work, we showed that thermal superradiance can be controlled meaningfully using a tuneable emitter array and our efforts pave the path to numerous possible applications, such as highly efficient photothermal cancer therapy devices.

\section{ACKNOWLEDGMENTS}

S.M. would like to thank M. P. N. Perera and members of $\mathrm{A} \chi \mathrm{L}$ at Monash University including D. Weeraddana, H. P. Hapuarachchi, and K. L. Chathuranga for encouragement and insightful discussions. The work of S.M. is supported by the Monash University Institute of Graduate Research, and the work of M.P. is supported by the Australian Research Council through its Discovery Grant No. DP140100383.
[1] R. H. Dicke, Phys. Rev. 93, 99 (1954).

[2] M. Gross and S. Haroche, Phys. Rep. 93, 301 (1982).

[3] S. John and T. Quang, Phys. Rev. Lett. 74, 3419 (1995).

[4] C. H. Keitel, M. O. Scully, and G. Süssmann, Phys. Rev. A 45, 3242 (1992).

[5] K. Higgins, S. Benjamin, T. Stace, G. Milburn, B. W. Lovett, and E. Gauger, Nat. Commun. 5, 4705 (2014).

[6] F. Haake, M. I. Kolobov, C. Fabre, E. Giacobino, and S. Reynaud, Phys. Rev. Lett. 71, 995 (1993).

[7] D. Meiser and M. J. Holland, Phys. Rev. A 81, 033847 (2010).

[8] D. Meiser and M. J. Holland, Phys. Rev. A 81, 063827 (2010).

[9] A. Auffèves, D. Gerace, S. Portolan, A. Drezet, and M. F. Santos, New J. Phys. 13, 093020 (2011).

[10] J. G. Bohnet, Z. Chen, J. M. Weiner, D. Meiser, M. J. Holland, and J. K. Thompson, Nature (London) 484, 78 (2012).

[11] H. M. Wiseman and G. J. Milburn, Quantum Measurement and Control (Cambridge University Press, Cambridge, UK, 2009).

[12] I. Celanovic, D. Perreault, and J. Kassakian, Phys. Rev. B 72, 075127 (2005).

[13] M. Kaviany, Heat Transfer Physics (Cambridge University Press, New York, 2014).

[14] E. Rephaeli and S. Fan, Opt. Express 17, 15145 (2009).

[15] X. Liu, T. Tyler, T. Starr, A. F. Starr, N. M. Jokerst, and W. J. Padilla, Phys. Rev. Lett. 107, 045901 (2011).

[16] K. A. Arpin, M. D. Losego, A. N. Cloud, H. Ning, J. Mallek, N. P. Sergeant, L. Zhu, Z. Yu, B. Kalanyan, G. N. Parsons et al., Nat. Commun. 4, 2630 (2013).
[17] B. J. Lee, C. J. Fu, and Z. M. Zhang, Appl. Phys. Lett. 87, 071904 (2005).

[18] M. De Zoysa, T. Asano, K. Mochizuki, A. Oskooi, T. Inoue, and S. Noda, Nat. Photonics 6, 535 (2012).

[19] M. Laroche, C. Arnold, F. Marquier, R. Carminati, J.-J. Greffet, S. Collin, N. Bardou, and J.-L. Pelouard, Opt. Lett. 30, 2623 (2005).

[20] M. S. Jang, V. W. Brar, M. C. Sherrott, J. J. Lopez, L. Kim, S. Kim, M. Choi, and H. A. Atwater, Phys. Rev. B 90, 165409 (2014).

[21] A. Narayanaswamy and G. Chen, Appl. Phys. Lett. 82, 3544 (2003).

[22] S. Shen, A. Narayanaswamy, and G. Chen, Nano Lett. 9, 2909 (2009).

[23] M. Zhou, S. Yi, T. S. Luk, Q. Gan, S. Fan, and Z. Yu, Phys. Rev. B 92, 024302 (2015).

[24] B.-Y. Hsieh and M. Jarrahi, J. Appl. Phys. 109, 084326 (2011).

[25] W. Suh, Z. Wang, and S. Fan, IEEE J. Quantum Electron. 40, 1511 (2004).

[26] H. A. Haus and W. Huang, Proc. IEEE 79, 1505 (1991).

[27] S. Fan, W. Suh, and J. D. Joannopoulos, J. Opt. Soc. Am. A 20 , 569 (2003).

[28] L. Zhu, S. Sandhu, C. Otey, S. Fan, M. B. Sinclair, and T. S. Luk, Appl. Phys. Lett. 102, 103104 (2013).

[29] H. A. Haus, Electromagnetic Noise and Quantum Optical Measurements (Springer-Verlag, Berlin, 2000). 
[30] L. Verslegers, Z. Yu, P. B. Catrysse, and S. Fan, J. Opt. Soc. Am. B 27, 1947 (2010).

[31] M. Premaratne and M. I. Stockman, Adv. Opt. Photonics 9, 79 (2017).

[32] F. J. Garcia de Abajo and J. J. Sáenz, Phys. Rev. Lett. 95, 233901 (2005).

[33] H. Cao and A. Nahata, Opt. Express 12, 1004 (2004).

[34] J. Gómez Rivas, C. Schotsch, P. Haring Bolivar, and H. Kurz, Phys. Rev. B 68, 201306 (2003).

[35] K. S. Miller, Math. Mag. 54, 67 (1981).

[36] H. E. Rose, Linear Algebra: A Pure Mathematical Approach (Springer Science \& Business Media, Berlin, 2002).

[37] Z. Yu, A. Raman, and S. Fan, Phys. Rev. Lett. 109, 173901 (2012).

[38] S. D. Gunapala, S. V. Bandara, J. K. Liu, E. M. Luong, S. B. Rafol, J. M. Mumolo, D. Z. Ting, J. J. Bock, M. E. Ressler, M. W. Werner et al., in AeroSense 2000 (SPIE, Bellingham, 2000), pp. 262-275.

[39] G. H. Döhler, H. Künzel, and K. Ploog, Phys. Rev. B 25, 2616 (1982).

[40] L. Verslegers, Z. Yu, Z. Ruan, P. B. Catrysse, and S. Fan, Phys. Rev. Lett. 108, 083902 (2012).

[41] Y. Takakura, Phys. Rev. Lett. 86, 5601 (2001).

[42] J. Bravo-Abad, L. Martin-Moreno, and F. J. Garcia-Vidal, Phys. Rev. E 69, 026601 (2004).

[43] O. Mata-Mendez and J. Avendaño, J. Opt. Soc. Am. A 24, 1687 (2007).

[44] P. Meystre and M. Sargent, Elements of Quantum Optics (Springer Science \& Business Media, Berlin, 2013).
[45] M. Fox, Quantum Optics: An Introduction (Oxford University Press, Oxford, 2006).

[46] G. Baffou and R. Quidant, Laser Photonics Rev. 7, 171 (2013).

[47] M. Premaratne and G. P. Agrawal, Light Propagation in Gain Media: Optical Amplifiers (Cambridge University Press, New York, 2011).

[48] M. Premaratne, E. Premaratne, and A. J. Lowery, Opt. Express 13, 389 (2005).

[49] D. Weeraddana, M. Premaratne, S. D. Gunapala, and D. L. Andrews, Phys. Rev. B 94, 085133 (2016).

[50] C. S. Kumarasinghe, M. Premaratne, Q. Bao, and G. P. Agrawal, Sci. Rep. 5, 12140 (2015).

[51] D. Weeraddana, M. Premaratne, and D. L. Andrews, Phys. Rev. B 92, 035128 (2015).

[52] D. Sikdar, I. D. Rukhlenko, W. Cheng, and M. Premaratne, Nanoscale Res. Lett. 8, 142 (2013).

[53] C. Rupasinghe, I. D. Rukhlenko, and M. Premaratne, ACS Nano 8, 2431 (2014).

[54] C. Jayasekara, M. Premaratne, M. I. Stockman, and S. D. Gunapala, J. Appl. Phys. 118, 173101 (2015).

[55] C. Jayasekara, M. Premaratne, S. D. Gunapala, and M. I. Stockman, J. Appl. Phys. 119, 133101 (2016).

[56] J. A. Ali, W. B. Wang, M. Zevallos, and R. R. Alfano, Technol. Cancer Res. Treat. 3, 491 (2004).

[57] F. J. Garcia-Vidal, L. Martin-Moreno, T. Ebbesen, and L. Kuipers, Rev. Mod. Phys. 82, 729 (2010).

[58] T. T. N. Dung, N. Q. Buu, D. V. Quang, H. T. Ha, L. A. Bang, N. H. Chau, N. T. Ly, and N. V. Trung, J. Phys.: Conf. Ser. 187, 012054 (2009). 